INVESTIGACIONES

\title{
Un caso de estudio sobre conocimiento previo en tres universidades ecuatorianas: UC, UDA y UNAE
}

\author{
A case study on previous knowledge in three Ecuadorian universities: \\ UC, UDA and UNAE
}

\author{
José Manuel Castellano Gil ${ }^{a}$, Efstathios Stefos ${ }^{b}$, Mariana Sánchez ${ }^{c}$, \\ Kamila Torres Orellana ${ }^{\text {, Danilo Isaac Reiban Garnica }}{ }^{e}$

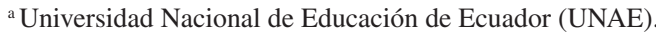 \\ Correo electrónico: jose.castellano@unae.edu \\ ${ }^{\mathrm{b}}$ Universidad Nacional de Educación de Ecuador (UNAE). \\ Correo electrónico: stefos.efstathios@unae.edu.ec. \\ ${ }^{\mathrm{c}}$ Universidad de Cuenca (UC). \\ Correo electrónico: mariana.sanchez@ucuenca.edu.ec. \\ ${ }^{\mathrm{d}}$ Universidad del Azuay (UDA.) \\ Correo electrónico: kamilat22@ hotmail.com. \\ e Universidad Nacional de Educación de Ecuador (UNAE). \\ Correo electrónico: daniloisaac305@gmail.com.
}

\begin{abstract}
RESUMEN
Este estudio es un intento de aproximación al estado previo y evolutivo del conocimiento de tres grupos de estudiantes universitarios adscriptos a la Universidad de Cuenca, la Universidad del Azuay y la Universidad Nacional de Educación de Ecuador. El eje central de este trabajo es explorar el punto de partida cognitivo del alumnado en aspectos esenciales de la asignatura en el momento inicial del semestre y su constatación a la finalización del mismo. Para alcanzar los objetivos reseñados se ha elaborado un instrumento articulado en cuatro módulos de contenidos y orientados desde una triple perspectiva analítica: a) identificar la característica inicial del grupo e individual; b) detectar los posibles cambios experimentados una vez concluido el semestre y c) valorar la incidencia del docente y ejercitar un proceso de reflexión del papel ejercido. Los resultados obtenidos en esta investigación muestran la existencia de cambios significativos en el proceso de aprendizaje.
\end{abstract}

Palabras Claves: Evaluación diagnóstica, conocimiento previo, estrategias didácticas.

\begin{abstract}
This study approximates the previous and evolutionary state of knowledge of three groups of university students attached to the University of Cuenca, the University of Azuay, and the National University of Education of Ecuador. The central focus of this work is to explore the starting point of the cognitive support of students in essential aspects of the subject at the beginning of the semester, and its verification at the end of the semester. In order to reach the objectives outlined, an instrument was elaborated in four content modules, oriented from a triple analytical perspective: a) to identify the initial characteristic of the group and individual; b) to detect and study the possible changes undergone by the students after the end of the semester; and c) to evaluate the teacher's impact and exercise a process of reflection and self-criticism of the role played. The results obtained in this research show the existence of significant changes in the learning of knowledge and in conceptual assimilation.
\end{abstract}

Keywords: Diagnostic evaluation, prior knowledge, didactic strategies. 


\section{INTRODUCCIÓN}

Una inquietud comúnmente compartida por el profesorado universitario es la brecha existente entre el conocimiento adquirido por los estudiantes durante la etapa de Bachillerato y lo que se requiere en su formación universitaria (Villegas 2015), así como las debilidades formativas detectadas en los alumnos en los distintos niveles de la enseñanza superior (Bonilla et al. 2017). Ese desfase afecta tanto a la base de conocimientos como a la formación básica en habilidades de aprendizaje (López 2009).

Este estudio, sin embargo, no pretende dar respuesta a esa premisa sino que intenta detectar los conocimientos previos de un grupo de alumnos, en el momento en que inician su semestre y constatar su evolución a la finalización del mismo periodo. Su propósito no se reduce exclusivamente a conocer el punto de partida de los conocimientos previos y su dinámica sino que tiene como objeto obtener indicadores con los que el docente pueda, en su caso, activar estrategias pedagógicas adecuadas que fomenten un aprendizaje significativo.

Desde el ámbito conceptual se denomina "ideas o conocimientos previos" a aquellas concepciones que los estudiantes han adquirido sobre diferentes conocimientos, tanto los construidos a partir de experiencias externas a la enseñanza sistemática como los creados en los procesos educativos (Rayas 2004). El procedimiento empleado para recopilar y abordar el estado de conocimiento ha sido a través de una evaluación diagnóstica, aplicada antes de iniciar la nueva fase de aprendizaje con la doble finalidad de saber qué sabe el alumno con respecto a lo que se le requiere y adoptar decisiones que fortalezcan su proceso educativo (Restrepo et al. 2014).

Los estudios centrados en este tipo de análisis tienen sus antecedentes en el último tercio de la pasada centuria (Carretero y Limón 1997). No obstante, ese enfoque en el ámbito educativo surge a partir de Bartlett y Kelly (Bartlett 1932; Kelly 1955), aunque no sería hasta la teoría de Ausubel, sobre el concepto de "aprendizaje significativo" y la importancia de los conocimientos previos, cuando comienza a adquirir un amplio desarrollo, como fundamento de la significatividad en el aprendizaje (Ausubel 1963). Posteriormente, otros autores, Viennot y Novak, abordaron estudios similares donde subrayan que los alumnos, antes de acceder a la instrucción formal, desarrollan ideas que prevalecen tras la fase de la enseñanza reglada (Viennot 1976; Novak 1982). Sin embargo, como se recogen en distintas aportaciones, no siempre esos conocimientos previos son obstáculos epistemológicos que dificultan la instrucción, pues, en determinadas ocasiones, simplemente representan un conocimiento incompleto o erróneo (Limón y Carretero 1996). En ambas situaciones el acompañamiento del docente parece ser clave para intentar revertir ese panorama, mediante la introducción de diversos mecanismos e instrumentos, siempre y cuando se disponga de información al respecto. En esa dirección se encamina esta propuesta: chequear los conocimientos previos del alumnado sobre la materia en la fase inicial y final del curso académico y que permitirá un ejercicio autoreflexivo sobre la propia práctica docente.

En estas últimas décadas se ha generado una rica producción científica, que ha superado la caracterización descrita por Carretero y Limón hace veinte años atrás: "el conocimiento de los «conocimientos previos» de los estudiantes sobre muchas áreas del currículum es todavía un tanto pobre y limitado" (Carretero y Limón 1997). Así, desde diferentes enfoques, desde diversas materias y sobre distintos niveles educativos se han elaborado destacados estudios centrados en el análisis de las ideas o conocimientos previos en la enseñanza. 
En la actualidad existe un consenso generalizado sobre el relevante papel que juegan los conocimientos previos en el proceso de aprendizaje y su participación en la construcción de nuevos significados, que "es tanto más significativo cuantas más relaciones con sentido es capaz de establecer el alumno entre lo que ya conoce, sus conocimientos previos y el nuevo contenido" (Coll 2004). Esa estrecha interrelación ha llevado a López a subrayar que para promover el aprendizaje significativo se debe considerar los conocimientos factuales, conceptuales, actitudinales y procedimentales y su conexión con la nueva información asimilada por los alumnos mediante los materiales de aprendizaje o las explicaciones del docente (López 2009). De modo que los conocimientos previos del alumnado constituyen un elemento de interés en la concepción constructivista, pero no desde un ángulo estático sino desde una perspectiva dinámica que intenta valorizar su incidencia en los procesos de enseñanza-aprendizaje. En ese sentido Ausubel, ya en su obra Psicología Educativa (Ausubel 1968) señalaba que "si tuviera que reducir toda la psicología educativa a un solo principio: El factor más importante que influye en el aprendizaje es lo que el alumno ya sabe. Averígüese esto y enséñele en consecuencia” (Novack 1987).

La vía de acceso a los conocimientos previos del alumnado en el contexto de los nuevos aprendizajes exige responder al qué, cuándo y cómo se interrelacionan junto a la necesidad de que sean evaluados. Ese diagnóstico inicial permite una aproximación real sobre su estado, ayuda a rediseñar la actuación docente a partir de la introducción y aplicación, como son, entre otros, los «organizadores previos» (Moreira 2008: 2; Pimienta 2012). Este planteamiento mantiene todavía un carácter innovador en el ámbito educativo actual, ya que supone una ruptura con respecto a las prácticas tradicionales.

El ejercicio de apropiación de las ideas previas por parte del profesor no está muy generalizado en las aulas y, por lo común, estos procesos de aprendizaje y la labor docente parten de otras ideas previas del profesor y no de las de los propios alumnos (Rayas 2004). Ese contexto propicia, en muchas ocasiones, un insalvable obstáculo entre el aprendiz y el maestro. Por el contrario esa apropiación, por parte del docente del conocimiento previo que poseen los alumnos proporcionan una fuente de información de gran peso y un indicador consistente no sólo para conocer la situación de partida, secuencia de prerrequisitos de aprendizaje, y las posibles falencias sino que permite actuar en la cimentación del proceso de aprendizaje y posibilita una intervención inmediata a través de diferentes actividades de aprendizaje (Solaz y Sanjosé 2008). De modo que el conocimiento previo se convierte en una variable a tener muy en cuenta en el nuevo aprendizaje y, por consiguiente, se hace imprescindible evaluar lo que sabe el alumno en el momento de iniciar una nueva etapa de aprendizaje.

\section{MATERIALES Y MÉTODOS}

La necesidad de disponer de datos, sobre el conocimiento previo de los alumnos en el proceso de adquisición y construcción de nuevos conceptos, ha llevado a diversas propuestas técnicas de diagnóstico ${ }^{1}$. En este estudio de caso que se presenta se ha optado por un diseño

\footnotetext{
Desde los primeros momentos los instrumentos utilizados estaban conformados por los test, entrevistas clínicas y las técnicas del mapa conceptuales (Novack 1987: 219). Posteriormente se han desarrollados diversas modalidades de cuestionarios, entre ellos el cuestionario denominado KPSI (Knowledge and Prior Study Inventory) diseñado por Tamir y Lunetta (Martínez 2015: 92); ejercicios de resolución de problemas (Solaz et al. 1995: s/p); procesos de compresión lectora (Kampouropoulou-Savvaidou et al 2014: 255) y estudios de observación en el aula (Papapostolou et al 2013: 89).
} 
cuasi-experimental pre-test/pos-test con grupos constituidos y, por tanto, no seleccionados de forma aleatoria (Restrepo et al. 2014) y por la elaboración de un cuestionario mixto, que integra preguntas abiertas ${ }^{2}$, cerradas junto a otras de opciones múltiples. Este instrumento tiene la visión de profundizar más allá del conocimiento propiamente académico con la pretensión de valorar una formación integral del alumnado, a través de la indagación sobre otros elementos significativos, cuya información ayuda a esbozar un perfil más completo que pueda servir de orientación al docente para un mejor desempeño de su labor.

Desde esa perspectiva se ha confeccionado un cuestionario que tiene como objeto evaluar el punto de partida de un grupo de alumnos en tres universidades ecuatorianas. El ámbito de esta investigación se centra en el estudio de elementos conceptuales básicos de la materia: en un primer momento, al inicio del curso, se aplica el instrumento propuesto y, en una etapa posterior, a la finalización del semestre, se repite su aplicación, aunque con la introducción de un único cambio en una de sus variables (Stefos \& Koulianidi 2016a:12). De esta manera se puede observar la evolución grupal e individual y valorar la incidencia ejercida por el docente.

El cuestionario está estructurado en tres módulos de contenidos con sus variables de estudio correspondiente. El primer módulo, datos identificativos, se dedica a determinadas características sociodemográficas del alumnado: provincia y cantón de nacimiento y residencia actual, titularidad jurídica de la unidad educativa donde cursó la Enseñanza Básica. Las características de clasificación personal utilizadas se reducen a la edad, género, estado civil, cargas familiares y autoreconocimiento étnico.

Un segundo grupo de variables explora el motivo de elección de la carrera de Educación, su conocimiento sobre el modelo pedagógico de su centro universitario, sus expectativas y valoración de la asignatura (Papapostolou \& Stefos 2013: 249).

El tercer módulo analiza las variables de conocimiento, conceptos generales sobre la materia y otros relacionados con el proceso de investigación (Stefos \& Koulianidi 2016b: 61).

La recogida de información se realizó en horario de clases y aplicada durante el semestre marzo-julio 2017 a dos paralelos de Historia de Vida del Segundo Ciclo de la carrera de Educación Básica de la UNAE, dos paralelos del Quinto Ciclo de la carrera de Turismo de la Facultad de Ciencias de Hospitalidad de la Universidad de Cuenca, correspondiente a la asignatura de "Ética, Derechos Humanos y Ciudadanía" y a un paralelo de Cuarto Ciclo de la Facultad de Filosofía, Letras y Ciencias de la Educación, Escuela de Educación Básica y Especial, en la materia: "Estudios Sociales II y Didáctica de la Universidad del Azuay.

Los estudiantes respondieron a las mismas variables dos veces con el fin de identificar la existencia de diferencia significativa entre ambas etapas (Stefos et al. 2011: 93). En el siguiente apartado se presentan los resultados del análisis descriptivo porcentual por variable y se ha aplicado también la prueba estadística $t$ para las muestras relacionadas con la finalidad de identificar diferencias significativas entre las respuestas a los dos cuestionarios. Para el análisis estadístico se utilizó el software R v.3.2.5.

El carácter abierto de las preguntas exigió una evaluación interpretativa de las respuestas y su clasificación en: adecuada, parcialmente adecuada e inadecuada (García \& Acevedo, 2016: 588). 


\section{RESULTADOS Y DISCUSIÓN}

Primer módulo: Caracterización de la población estudiantil analizada

El origen territorial del conjunto de los alumnos investigados de las tres universidades objeto de estudio tiene una procedencia de 12 de las 24 provincias ecuatorianas, siendo las provincias donde se ubican los centros universitarios y su limítrofe (Azuay o Cañar) las que aportan un mayor nivel de representación estudiantil. De este modo el 77\%, 70\% y el $43 \%$ respectivamente de los universitarios del Azuay, Cuenca y UNAE tienen como lugar de nacimiento la provincia de Azuay. Mientras que el 15\%, 18\% y 31\% se corresponde con la provincia de El Cañar. Estos datos indican que 3/4 parte de la población estudiantil analizada de esas universidades se nutren básicamente de su propio entorno territorial o de la provincia más próxima. Por tanto, la aportación de las restantes provincias ecuatorianas presenta un bajo nivel porcentual, a excepción de la UNAE que alcanza un $26 \%$.

Tabla 1: Distribución porcentual por provincia de nacimiento.

\begin{tabular}{|l|c|c|c|}
\hline Provincias & UNAE \% & U. CUENCA \% & UDA \% \\
\hline Azuay & 43 & 70 & 77 \\
\hline Cañar & 31 & 18 & 0 \\
\hline Sucumbíos & 4 & 0 & 0 \\
\hline Pichincha & 4 & 2 & 0 \\
\hline Guayas & 4 & 2 & 0 \\
\hline Cotopaxi & 4 & 0 & 0 \\
\hline Imbabura & 4 & 0 & 0 \\
\hline Morona Santiago & 2 & 0 & 0 \\
\hline Santa Elena & 2 & 0 & 0 \\
\hline Manabí & 2 & 0 & 8 \\
\hline Loja & 0 & 2 & 100 \\
\hline El Oro & 100 & 60 & 0 \\
\hline Total & & & 0 \\
\hline
\end{tabular}

El universo de estudio de los alumnos de la UNAE, compuesto por 49 alumnos, está distribuido por un $49 \%$ de mujeres y un $51 \%$ de hombres y sus franjas etarias las hemos agrupadas en dos intervalos: un 59\% se encuentra entre los 18 a 22 años y un $41 \%$ tienen 23 o más años, siendo su media de 22 años. Un dato que nos habla de una elevada edad para ser alumnos del segundo semestre universitario.

En relación al universo de estudio de los alumnos adscriptos a la Universidad de Cuenca que cursan quinto semestre, integrado por 44 estudiantes, tiene una distribución 
por género equilibrada, un $50 \%$ de mujeres y un $50 \%$ de hombres, con un intervalo de edades de 18 a 22 años que representa un 59\% y un grupo de más de 23 años que alcanza un $41 \%$, con una media de edad de $21,59 \%$.

Con respecto al universo de los estudiantes de la Universidad del Azuay, compuesto por 13 alumnos de cuarto semestre, presenta una mayoría de mujeres que asciende a un $77 \%$ frente a un $23 \%$ de varones y donde un 54\% tienen entre 18 a 22 años y un $46 \%$ más de 23 años, con una media de 21,84 años.

Otro rasgo distintivo se aprecia en el carácter jurídico de las unidades educativas de procedencia de los estudiantes. Así en la UNAE un $78 \%$ de los miembros analizados habían cursado sus estudios de Educación Básica en unidades educativas fiscales, es decir públicas, mientras que un $14 \%$ proceden de centros particulares y un $8 \%$ de instituciones fiscomisionales (colegios particulares financiados por el Gobierno ecuatoriano). Una tendencia similar presenta la UDA, aunque casi duplica el porcentaje de alumnos procedentes de centros particulares $(23 \%)$ frente a la UNAE. Mientras que la U. de Cuenca ofrece una evolución diferente, caracterizada por un equilibrio porcentual entre los centros públicos y particulares, con un $48 \%$ y $45 \%$ respectivamente.

Tabla 2: Distribución porcentual del carácter jurídico de los centros educativos de procedencia.

\begin{tabular}{|l|c|c|c|}
\hline Régimen jurídico & UNAE & U. CUENCA & UDA \\
\hline Fiscal & 78 & 48 & 69 \\
\hline Particular & 14 & 45 & 23 \\
\hline Fiscomisional & 8 & 7 & 8 \\
\hline Total & 100 & 100 & 100 \\
\hline
\end{tabular}

En relación al índice sobre el estado civil de los estudiantes de la U. de Cuenca, UDA y la UNAE se aprecia un elevado porcentaje de solteros, que se sitúan en un 93\%, $92 \%$ y $88 \%$ respectivamente. Sin embargo entre un $8 \%$ al $10 \%$ de total de esos alumnos cuentan con cargas familiares (véase tablas 4 y 5 ).

Tabla 3: Distribución porcentual del estado civil del alumnado.

\begin{tabular}{|l|c|c|c|}
\hline Estado civil & UNAE & U. CUENCA & UDA \\
\hline Soltero/a & 88 & 93,18 & 92 \\
\hline Casado/a & 6 & 2,27 & 8 \\
\hline Unión libre & 4 & 2,27 & 0 \\
\hline Separado/a & 2 & 2,27 & 0 \\
\hline Total & 100 & 100 & 100 \\
\hline
\end{tabular}


Tabla 4: Distribución porcentual de alumnos con hijos.

\begin{tabular}{|l|c|c|c|}
\hline Hijos/as & UNAE & U. CUENCA & UDA \\
\hline $\mathrm{Si}$ & 10 & 9 & 8 \\
\hline No & 90 & 91 & 92 \\
\hline Total & 100 & 100 & 100 \\
\hline
\end{tabular}

Tabla 5: Distribución porcentual del número de hijos.

\begin{tabular}{|l|c|c|c|}
\hline Hijos/as & UNAE & U. CUENCA & UDA \\
\hline 0 & 90 & 91 & 92 \\
\hline 1 & 6 & 9 & 8 \\
\hline 2 & 4 & 0 & 0 \\
\hline Total & 100 & 100 & 100 \\
\hline
\end{tabular}

Desde el punto de vista del autoreconocimiento étnico, el $98 \%$ tanto de los alumnos de la UNAE y la U. de Cuenca y un $92 \%$ de la UDA se definen como mestizo. Mientras que un $2 \%$ de la UNAE se considera indígena, un porcentaje similar en la U. de Cuenca como mulato y un $8 \%$ de la UDA como indígena.

Tabla 6: Distribución porcentual de autoreconocimiento étnico del alumnado.

\begin{tabular}{|l|c|c|c|}
\hline Etnia & UNAE & U. CUENCA & UDA \\
\hline Mestizo & 98 & 98 & 92 \\
\hline Indígena & 2 & 0 & 8 \\
\hline Mulato & 0 & 2 & 0 \\
\hline Afroecuatoriano & 0 & 0 & 0 \\
\hline Montubio & 0 & 0 & 0 \\
\hline Blanco & 0 & 0 & 0 \\
\hline Total & 100 & 100 & 100 \\
\hline
\end{tabular}


Estudios Pedagógicos XLIV, $\mathrm{N}^{\circ}$ 1: 377-402, 2018

UN CASO DE ESTUDIO SOBRE CONOCIMIENTO PREVIO EN TRES UNIVERSIDADES ECUATORIANAS: UC, UDA Y UNAE

Segundo módulo: Motivación en la elección de la carrera, conocimiento del modelo pedagógico, expectativas de la asignatura y valoración

La motivación a la hora de elegir una carrera de Educación Superior es, sin duda, un dilema para la gran parte de los futuros alumnos universitario y constituye un indicador complementario a considerar. De los resultados obtenidos llama la atención dos aspectos relevantes: por un lado, la inmensa mayoría del alumnado objeto de nuestra atención se ajusta a los criterios y valores propios que deben primar en la elección de convertirse en profesionales de sus ámbitos y, en segundo lugar, se observa una clara correspondencia entre las respuestas ofrecidas en el test previo y posterior, como se puede apreciar en las tablas 7, 8, y 9.

Tabla 7: Motivación en la elección de la carrera de Educación (UNAE).

\begin{tabular}{|l|c|c|}
\hline & Pre-test \% & Pos-test $\%$ \\
\hline Vocacional & 14 & 14 \\
\hline Preferencias por materias específicas (literatura y matemáticas) & 2 & 2 \\
\hline Experiencia vivida como tutor & 6 & 6 \\
\hline Le gusta trabajar con los niños & 16 & 16 \\
\hline Formar y transmitir conocimientos & 14 & 14 \\
\hline Mejorar la educación y fomentar cambios & 20 & 20 \\
\hline Condicionado (no tenía otra alternativa) & 6 & 8 \\
\hline Afinidad a la UNAE & 6 & 8 \\
\hline Tradición familiar & 10 & 10 \\
\hline NA & 4 & 0 \\
\hline Total & 100 & 100 \\
\hline
\end{tabular}

Tabla 8: Motivación en la elección de la carrera de Turismo (U. CUENCA).

\begin{tabular}{|l|c|c|}
\hline & Pre-test \% & Post-test $\%$ \\
\hline Vocación & 14 & 18 \\
\hline Turismo es una carrera que tiene futuro en el Ecuador & 14 & 7 \\
\hline Conocer destinos turísticos & 30 & 36 \\
\hline Aprender sobre cultura e historia & 18 & 20 \\
\hline Aprender diferentes idiomas & 7 & 7 \\
\hline No tenía otra opción & 5 & 2 \\
\hline Llegar a ser azafata & 5 & 5 \\
\hline NA & 9 & 5 \\
\hline Total & 100 & 100 \\
\hline
\end{tabular}


Tabla 9: Motivación en la elección de la carrera de Educación Básica y Especial (UDA).

\begin{tabular}{|l|c|c|}
\hline & Pre-test $\%$ & Post-test $\%$ \\
\hline Vocación & 8 & 8 \\
\hline Le gusta trabajar con niños & 15 & 23 \\
\hline Ayudar a las personas discapacitadas & 8 & 23 \\
\hline Seguir ejemplo familiar & 8 & 8 \\
\hline Mejorar la educación y fomentar cambios & 46 & 38 \\
\hline NA & 15 & 0 \\
\hline Total & 100 & 100 \\
\hline
\end{tabular}

Desde otro ángulo este módulo intenta detectar el conocimiento previo que el alumnado posee sobre el Modelo Pedagógico de sus respectivos centros a través de dos ítems: uno cerrado, donde se pregunta "si conoce" o "no" el modelo y otro abierto, donde se solicita una demostración por escrito sobre su capacidad al respecto con la idea de verificar la respuesta anterior.

En la fase del pre-test un $76 \%$ de los alumnos de la UNAE responden que conoce el Modelo Pedagógico y en el pos-test ese resultado se incrementaba hasta un $90 \%$. De modo, y a pesar del alto conocimiento inicial, esta variable experimenta un cambio altamente significativo, pues el valor proporcionado por la prueba estadística $t$ se sitúa en el 0,018.

Con respecto a la Universidad de Cuenca el 100\% de los alumnos objeto de estudio afirman desconocer el modelo pedagógico durante la fase del pre-test, porcentaje éste que disminuye en 18 puntos en el pos-test, con lo que la prueba $t$ registra un índice de 0.003 , que señala un cambio significativo. Mientras que en la UDA un 23\% afirman conocer el modelo pedagógico en el pre-test, registrándose posteriormente un crecimiento de 31 puntos en el pos-test para alcanzar un 0.003 en la prueba $T$, que refleja asimismo un cambio significativo.

Tabla 10: Asimilación del modelo pedagógico.

\begin{tabular}{|l|c|c|c|c|c|c|}
\hline & \multicolumn{2}{|c|}{ UNAE } & \multicolumn{2}{c|}{ U. CUENCA } & \multicolumn{2}{c|}{ UDA } \\
\hline & $\mathrm{Si}$ & $\mathrm{No}$ & $\mathrm{Si}$ & $\mathrm{No}$ & $\mathrm{Si}$ & No \\
\hline Pre-test & 76 & 24 & 0 & 100 & 23 & 77 \\
\hline Pos-test & 90 & 10 & 18 & 82 & 54 & 46 \\
\hline
\end{tabular}

Con la idea de constatar esa información proporcionada por el alumnado se solicitaba, como hemos señalado, que definiera el modelo pedagógico. De esta manera se pudo observar que ni un solo estudiantes de la UNAE respondía adecuadamente durante el pre- 
test. En cambio en el pos-test un $41 \%$ contestaba de forma adecuada. Mientras que las respuestas parcialmente adecuadas se mantenían estables con un 53\%, tanto en el pretest como en el pos-test, y las respuestas inadecuadas descendían de un $47 \%$ al $6 \%$ en el pos-test. Esa evolución ofrece cambios altamente significativos confirmados por el índice obtenido en la prueba $t(0,000)$.

En relación a los alumnos de la Universidad de Cuenca los datos obtenidos en la fase del pre-test reflejan como el $100 \%$ de sus respuestas son inadecuadas, mientras que en el pos-test tan sólo un 7\% son parcialmente adecuadas. Y como resultante el índice obtenido en la prueba $t(0.083)$ muestra la inexistencia de cambios.

Con respecto a los alumnos de la Universidad del Azuay tan sólo un $8 \%$ ofrecían respuestas validas en el pre-test y un porcentaje similar correspondían a respuestas parcialmente adecuadas, mientras que un $85 \%$ fueron inadecuadas. Sin embargo el postest refleja un cambio de tendencia, pues un $46 \%$ respondían adecuadamente frente a un $54 \%$ inadecuada, alcanzando un índice de 0,044 en la prueba $t \mathrm{y}$, por tanto, confirmando un cambio significativo en este proceso de aprendizaje.

\section{Expectativa inicial sobre la materia}

Otro aspecto que también interesa conocer se centra en las expectativas generadas por el alumnado sobre la materia en el momento inicial del semestre, ya que consideramos que es una información complementaria a tener muy en cuenta por el docente. En este caso las principales expectativas apuntadas por los estudiantes de los tres centros universitarios fueron: "Formarse para aprender a investigar", "Mejoras destrezas y habilidad para un futuro profesional", "Seguir ampliando conocimientos", "Aprender", etc. Éstas y el conjunto de las expectativas reseñadas, que se pueden apreciar en las tablas 11, 12 y 13, indican, a nuestro juicio, un definido grado de madurez de los estudiantes.

Tabla 11: Distribución porcentual de las expectativas sobre la materia (UNAE).

\begin{tabular}{|l|c|}
\hline Expectativas & $\%$ \\
\hline Aprender para ponerlo en práctica & 18 \\
\hline Mejorar destrezas y habilidades para un futuro profesional & 27 \\
\hline Formarse para aprender a investigar & 37 \\
\hline Que la materia no sea aburrida & 10 \\
\hline Aprender pedagogía & 4 \\
\hline NA & 4 \\
\hline Total & 100 \\
\hline
\end{tabular}


Tabla 12: Distribución porcentual de las expectativas sobre la materia (Universidad de CUENCA).

\begin{tabular}{|l|c|}
\hline Expectativas & $\%$ \\
\hline Que no se muy teórica & 18 \\
\hline Seguir ampliando conocimientos & 30 \\
\hline Aprender sobre Ética & 23 \\
\hline Aprender sobre el comportamiento humano & 16 \\
\hline NA & 14 \\
\hline Total & 100 \\
\hline
\end{tabular}

Tabla 13: Expectativas sobre la materia (Universidad del Azuay).

\begin{tabular}{|l|c|}
\hline Expectativas & $\%$ \\
\hline Adquirir conocimientos sobre Ecuador y América Latina & 38 \\
\hline Aprender Historia & 46 \\
\hline NA & 15 \\
\hline Total & 100 \\
\hline
\end{tabular}

\section{Valoración sobre la impartición de la materia}

Si importante es acercarse al conocimiento sobre las expectativas que el alumnado dispone en los momentos iniciales, no menos relevante es conocer su valoración sobre el proceso de formación recibido a la finalización del ciclo. La evaluación realizada por el alumnado, como puede observarse en los siguientes cuadros, resulta positiva, aunque ello no debe evitar una profunda reflexión, implementación de estrategias, acciones e incentivación.

Tabla 14: Valoración sobre la impartición de la materia UNAE

\begin{tabular}{|l|c|}
\hline & $\%$ \\
\hline Excelente e interesante por todo lo aprendido & 18 \\
\hline Examinar el pasado y poner en el presente según las experiencias vividas & 14 \\
\hline Gran ayuda para seguir aprendiendo a investigar & 29 \\
\hline Materia que ayuda al perfeccionamiento como futuros docentes & 27 \\
\hline Faltó más guía para la realización de diferentes actividades & 4 \\
\hline NA & 8 \\
\hline Total & 100 \\
\hline
\end{tabular}


Tabla 15: Valoración sobre la impartición de la materia U. CUENCA

\begin{tabular}{|l|c|}
\hline & $\%$ \\
\hline Excelente e interesante por todo lo aprendido & 36 \\
\hline La ética es muy importante & 5 \\
\hline Transcendente para la formación profesional & 14 \\
\hline Gran ayuda para la cultura general & 14 \\
\hline NA & 32 \\
\hline Total & 100 \\
\hline
\end{tabular}

Tabla 16: Valoración sobre la impartición de la materia UDA

\begin{tabular}{|l|c|}
\hline & $\%$ \\
\hline Excelente e interesante por todo lo aprendido & 46 \\
\hline La materia ayudó a conocer más sobre historia & 38 \\
\hline NA & 15 \\
\hline Total & 100 \\
\hline
\end{tabular}

\section{Tercer módulo: Asimilación conceptual}

Universidad Nacional de Educación de Ecuador (UNAE)

Asimilación del concepto investigación-acción

El indicador obtenido sobre este concepto en la fase del pre-test ofrece una información preocupante, ya que tan sólo un $4 \%$ de los estudiantes demuestra poseer capacidad de conocimiento. Mientras que un $49 \%$ ofrece una respuesta parcialmente adecuada y un $47 \%$ no posee dominio conceptual. Sin embargo los datos proporcionados en el pos-test proporcionan un incremento en las respuestas adecuadas (55\%), un descenso de 45 puntos en las inadecuadas y una disminución de 6 puntos en las parcialmente adecuadas. Producto de esa evolución el índice obtenido en la prueba estadística $t(0.000)$ señala un cambio significativo en esta asimilación conceptual.

Tabla 17: Distribución porcentual sobre la asimilación del concepto investigación-acción UNAE

\begin{tabular}{|l|c|c|c|}
\hline & Adecuada & Parcialmente adecuada & Inadecuada \\
\hline Pre-test & 4 & 49 & 47 \\
\hline Pos-test & 55 & 43 & 2 \\
\hline
\end{tabular}




\section{Asimilación sobre los propósitos que persigue la investigación-acción}

El indicador de conocimientos sobre los propósitos de la investigación-acción en el pretest arroja también otro dato inquietante, ya que tan sólo el $2 \%$ responde adecuadamente. Mientras que las respuestas parcialmente adecuadas y las inadecuadas se distribuyen con un $63 \%$ y un $35 \%$ respectivamente.

En cambio el registro de esos datos en el pos-test dibuja una reducción significativa en las inadecuadas, de un $35 \%$ a un $6 \%$; un alto crecimiento en las adecuadas que alcanza el $33 \%$; y un descenso de dos puntos en las parcialmente adecuadas. De tal modo que el dato obtenido en la prueba estadística $t(0.000)$ señala un avance significativo en este proceso de aprendizaje.

Tabla 18: Distribución porcentual sobre la asimilación de los propósitos que persigue

la investigación-acción

\begin{tabular}{|l|c|c|c|}
\hline & Adecuada & Parcialmente adecuada & Inadecuada \\
\hline Pre-test & 2 & 63 & 35 \\
\hline Pos-test & 33 & 61 & 6 \\
\hline
\end{tabular}

\section{Asimilación sobre las fases de una investigación}

Este indicador de conocimiento sobre las fases de una investigación es la única variable donde el porcentaje obtenido en el pre-test ofrece un dato altamente positivo, ya que un $24 \%$ responde adecuadamente, al tiempo, que las respuestas inadecuadas se sitúan en un $47 \%$ y las parcialmente adecuadas representan un 29\%. Estos positivos porcentajes se mejoran en la fase posterior con un importante crecimiento en la asimilación que alcanza un $65 \%$, un descenso de once puntos en las respuestas parcialmente adecuadas y una baja de 31 puntos en las inadecuadas. De este modo la prueba $t(0.000)$ señala la existencia significativa de asimilación conceptual.

Tabla 19: Distribución porcentual sobre la asimilación de las fases de una investigación

\begin{tabular}{|l|c|c|c|}
\hline & Adecuada & Parcialmente adecuada & Inadecuada \\
\hline Pre-test & 24 & 29 & 47 \\
\hline Pos-test & 65 & 18 & 16 \\
\hline
\end{tabular}




\section{Asimilación sobre los conceptos de investigación cualitativa-cuantitativa}

La precariedad mostrada en el dominio conceptual de investigación cualitativa-cuantitativa queda evidenciada con una nula representación de las respuestas adecuadas, con un $61 \%$ de las parcialmente adecuadas y con un 39\% de inadecuadas en el pre-test. Sin embargo en la fase del pos-test se produce una transformación relevante, pues el 47\% de las respuestas son adecuadas, un $49 \%$ parcialmente adecuadas y un $4 \%$ inadecuadas. Esa evolución se traduce en el índice obtenido por la prueba $t(0.000)$ que confirma un cambio destacado de aprendizaje.

Tabla 20: Distribución porcentual sobre la asimilación de los conceptos investigación cualitativa-cuantitativa

\begin{tabular}{|l|c|c|c|}
\hline & Adecuada & Parcialmente adecuada & Inadecuada \\
\hline Pre-test & 0 & 61 & 39 \\
\hline Pos-test & 47 & 49 & 4 \\
\hline
\end{tabular}

\section{Asimilación sobre el concepto Historia de vida}

Los indicadores de conocimiento previo sobre el concepto de Historia de vida en el pre-test registran un bajo porcentaje de respuestas adecuadas, un 14\%, un 53\% de las parcialmente adecuadas y un $33 \%$ de inadecuadas. No obstante nuevamente se produce un cambio de tendencia en el pos-test, donde las respuestas adecuadas crecen 57 puntos, mientras que las parcialmente inadecuadas descienden 24 puntos y las inadecuadas se reducen en 33 puntos. De manera que el índice ofrecido por la prueba $t(0.000)$ apunta hacia un aprendizaje conceptual significativo.

Tabla 21: Distribución porcentual sobre la asimilación del concepto Historia de vida

\begin{tabular}{|l|c|c|c|}
\hline & Adecuada & Parcialmente adecuada & Inadecuada \\
\hline Pre-test & 14 & 53 & 33 \\
\hline Pos-test & 71 & 29 & 0 \\
\hline
\end{tabular}

\section{Asimilación sobre los instrumentos utilizados en la construcción de Historias de vida}

Esta variable presenta unos indicadores previos aceptables, con un $31 \%$ de respuestas adecuadas, un $41 \%$ parcialmente adecuadas y un $29 \%$ inadecuadas. Mientras que su tendencia en el pos-test indica un avance sustancial de las respuestas adecuadas que crecen 40 puntos, un descenso de 17 puntos en las parcialmente adecuadas y una disminución 
de 25 puntos en las inadecuadas. Producto de esa evolución la prueba $t(0.000)$ viene a confirmar la existencia de un cambio significativo en su asimilación conceptual.

Tabla 22: Distribución porcentual sobre la asimilación de instrumentos utilizados en la construcción de historias de vida

\begin{tabular}{|l|c|c|c|}
\hline & Adecuada & Parcialmente adecuada & Inadecuada \\
\hline Pre-test & 31 & 41 & 29 \\
\hline Pos-test & 71 & 24 & 4 \\
\hline
\end{tabular}

\section{Asimilación del concepto hipótesis}

Este es otro de los indicadores que en la fase del pre-test muestran unos índices de escaso dominio conceptual, pues tan sólo $8 \%$ de las respuestas son adecuadas, un 65\% parcialmente inadecuadas y un $25 \%$ inadecuadas. De igual modo que en los casos anterior, los resultados obtenidos en el post-test vienen a registra un crecimiento de 23 puntos en las acertadas, una disminución de 10 puntos en las parcialmente adecuadas y un descenso de 13 puntos en las inadecuadas. De modo que el valor arrojado por la prueba $t(0.000)$ verifica un cambio significativo en el aprendizaje.

Tabla 23: Distribución porcentual sobre la asimilación del concepto hipótesis

\begin{tabular}{|l|c|c|c|}
\hline & Adecuada & Parcialmente adecuada & Inadecuada \\
\hline Pre-test & 8 & 65 & 27 \\
\hline Pos-test & 31 & 55 & 14 \\
\hline
\end{tabular}

\section{Universidad de Cuenca (UC)}

Asimilación del concepto ética

Un $18 \%$ de los alumnos de la U. de Cuenca demuestra su dominio previo cognitivo sobre el conceptual ética, mientras que un $50 \%$ de las respuestas son parcialmente adecuados y un $23 \%$ inadecuadas. Sin embargo, a pesar de un crecimiento de 14 puntos en el pre-test de las adecuadas, se aprecia un leve descenso de 5 puntos en las parcialmente adecuadas y una disminución de 9 puntos en las inadecuadas. El valor obtenido en la prueba $t(0.142)$ señala la inexistencia de cambio significativo. 
Tabla 24: Distribución porcentual sobre la asimilación del concepto Ética (U. CUENCA)

\begin{tabular}{|l|c|c|c|}
\hline & Adecuada & Parcialmente adecuada & Inadecuada \\
\hline Pre-test & 18 & 50 & 32 \\
\hline Pos-test & 32 & 45 & 23 \\
\hline
\end{tabular}

\section{Asimilación del concepto utilitarismo}

Esta variable en el pre-test muestra porcentajes muy bajos en las respuestas adecuadas y parcialmente adecuados, entre un 5\% y $7 \%$ respectivamente, y destaca en sobremanera las inadecuadas con un $89 \%$. Esa tendencia se invierte en el pos-test, donde las adecuadas se incrementan en 12 puntos, las parcialmente adecuadas se duplican y se produce un descenso de 21 puntos en las inadecuadas, que lleva a situar el índice de la prueba $t$ en $0.012 \mathrm{y}$, por consiguiente, refleja la existencia de un cambio significativo en su aprendizaje.

Tabla 25: Distribución porcentual sobre la asimilación conceptual de utilitarismo (U. CUENCA)

\begin{tabular}{|l|c|c|c|}
\hline & Adecuada & Parcialmente adecuada & Inadecuada \\
\hline Pre-test & 5 & 7 & 89 \\
\hline Pos-test & 18 & 14 & 68 \\
\hline
\end{tabular}

\section{Asimilación conceptual de los Derechos Humanos}

Los porcentajes obtenidos sobre la asimilación conceptual de Derechos Humanos ponen en evidencia unos resultados muy positivos en el pre-test, pues las respuestas adecuadas alcanzan el 50\%, las parcialmente adecuadas se sitúan en un 39\% y las inadecuadas un 5\%. Este cuadro mejora sustancialmente en el pos-test donde se aprecia un crecimiento de 30 puntos en las adecuadas, una disminución de 21 puntos en las parcialmente adecuadas y tres puntos en las inadecuadas. Asimismo el índice resultante de la prueba $t(0.001)$ apunta hacia un cambio altamente significativo.

Tabla 26: Distribución porcentual sobre la asimilación conceptual de los Derechos Humanos (U. CUENCA)

\begin{tabular}{|l|c|c|c|}
\hline & Adecuada & Parcialmente adecuada & Inadecuada \\
\hline Pre-test & 50 & 39 & 5 \\
\hline Pos-test & 80 & 18 & 2 \\
\hline
\end{tabular}




\section{Asimilación conceptual de ciudadanía}

Con respecto a la asimilación del concepto ciudadanía un $16 \%$ responden adecuadamente en el pre-test y se registra un crecimiento de 23 puntos en la fase final del semestre que manifiesta un cambio significativo (0.010), aunque se aprecia la permanencia de un porcentaje elevado en las respuestas inadecuadas en el pos-test (32\%).

Tabla 27: Distribución porcentual sobre la asimilación conceptual de ciudadanía (U. CUENCA)

\begin{tabular}{|l|c|c|c|}
\hline & Adecuada & Parcialmente adecuada & Inadecuada \\
\hline Pre-test & 16 & 41 & 43 \\
\hline Pos-test & 39 & 30 & 32 \\
\hline
\end{tabular}

\section{Conocimiento sobre la existencia de un Código ético en la UC}

Otro aspecto a analizar se centraba en detectar el conocimiento de los alumnos sobre la existencia de un Código ético de la Universidad. Y en ese sentido un $18 \%$ aseguraba conocer frente a un $82 \%$ que desconocían en el momento de aplicación del pre-test. Esa diferencia importante se atenúa en el pre-test, que da lugar un equilibrio porcentual entre ambas opciones al tiempo que se aprecia un cambio significativo (0.002), aunque el índice de los alumnos que continúan desconociendo su existencia sea bastante elevado (48\%).

Tabla 28: Distribución porcentual sobre el conocimiento de un Código Ético en la Universidad de Cuenca.

\begin{tabular}{|l|c|c|}
\hline & Si & No \\
\hline Pre-test & 18 & 82 \\
\hline Pos-test & 52 & 48 \\
\hline
\end{tabular}

Origen de la adquisición de conocimiento de un Código ético UC

Relacionado con el ítem anterior se pretendía indagar sobre la fuente de adquisición de ese conocimiento por parte del alumnado. Un primer elemento a resaltar es que los datos reflejan una concordancia con la tabla anterior y que un $18 \%$ y $52 \%$, respectivamente en el pre-test y pos-test, manifiestan que fue el aula el punto de origen de acceso a ese conocimiento. Esa evolución, independientemente del alto porcentaje de los que NA, refleja asimismo un cambio significativo (0.000). 
Tabla 29: Distribución porcentual sobre el origen de adquisición de conocimiento de la existencia de un Código Ético en la Universidad de Cuenca.

\begin{tabular}{|l|c|c|}
\hline & En aula & NA \\
\hline Pre-test & 16 & 84 \\
\hline Pos-test & 52 & 48 \\
\hline
\end{tabular}

\section{Asimilación conceptual de una teoría ética}

Los resultados de esta variable arrojan, en ambas fases, la existencia de un porcentaje elevado de dominio previo sobre alguna de las teorías ética, al tiempo que indica un leve crecimiento de 7 puntos entre los dos períodos (0.372).

Tabla 30: Distribución porcentual sobre la asimilación conceptual de una teoría ética, U. CUENCA

\begin{tabular}{|l|c|c|c|}
\hline & Adecuada & Parcialmente adecuada & Inadecuada \\
\hline Pre-test & 61 & 0 & 39 \\
\hline Pos-test & 68 & 0 & 32 \\
\hline
\end{tabular}

\section{Universidad del Azuay (UDA)}

\section{Asimilación conceptual de América Latina}

Los resultados obtenidos en el pre-test evidencian que el 69\% de las respuestas fueron inadecuadas con una distribución equilibrada entre las adecuadas y las parcialmente adecuadas que alcanzan entre ambas el $30 \%$. No obstante esa tendencia se invierte en el pos-test donde las respuestas adecuadas se incrementan en 31 puntos, las parcialmente adecuadas mejoran en 8 puntos y las inadecuadas se reducen en 38 puntos. Producto de esa evolución el registro que ofrece la prueba $t(0.032)$ refleja un cambio significativo en la asimilación por parte de los estudiantes en este concepto.

Tabla 31: Distribución porcentual sobre la asimilación del conceptual de América Latina (UDA)

\begin{tabular}{|l|c|c|c|}
\hline & Adecuada & Parcialmente adecuada & Inadecuada \\
\hline Pre-test & 15 & 15 & 69 \\
\hline Pos-test & 46 & 23 & 31 \\
\hline
\end{tabular}




\section{Asimilación cognitiva de la problemática actual en América Latina}

Esta variable ofrece unos mejores porcentajes de conocimiento previo en el pre-test ya que los porcentajes entre respuestas adecuadas y las parcialmente adecuadas ascienden al $69 \%$ frente a las inadecuadas que obtienen un $31 \%$. Estos resultados mejoran considerablemente en el cuestionario de fin de semestre al producirse un incremento en las respuestas adecuadas de 62 puntos, una disminución de 38 puntos en las parcialmente adecuadas y una reducción de 23 puntos en las inadecuadas. Estos excelentes resultados se reflejan en el índice proporcionado por la prueba $t(0.002)$ que viene a confirmar un cambio significativo de aprendizaje.

Tabla 32: Distribución porcentual sobre el conocimiento de la problemática actual en América Latina (UDA)

\begin{tabular}{|l|c|c|c|}
\hline & Adecuada & Parcialmente adecuada & Inadecuada \\
\hline Pre-test & 23 & 46 & 31 \\
\hline Pos-test & 85 & 8 & 8 \\
\hline
\end{tabular}

\section{Asimilación del conceptual de la teoría de dependencia}

Los resultados obtenidos en esta variable, tanto en la fase de pre-test como en la del postest, arrojan unos datos muy precarios, pues en ambas pruebas los porcentajes de respuestas inadecuadas se sitúan entre un $92 \%$ y un $69 \%$. De modo que el índice de la prueba $t(0.165)$ evidencia la inexistencia de cambio significativo en el aprendizaje.

Tabla 33: Distribución porcentual sobre la asimilación conceptual de la teoría de dependencia (UDA)

\begin{tabular}{|l|c|c|c|}
\hline & Adecuada & Parcialmente adecuada & Inadecuada \\
\hline Pre-test & 0 & 8 & 92 \\
\hline Pos-test & 8 & 23 & 69 \\
\hline
\end{tabular}

\section{Asimilación del conceptual sobre diversidad en América Latina}

Una evolución bien distinta presenta esta variable que se manifiesta con un fuerte crecimiento de 46 puntos con respecto a las respuestas adecuadas, un ligero descenso de 7 puntos en las parcialmente adecuadas y un brusco descenso de 40 puntos en las inadecuadas, que se ve reflejada en el resultado de la prueba $t(0.002)$ que confirma un cambio significativo. 
Tabla 34: Distribución porcentual sobre la asimilación conceptual diversidad en América Latina (UDA)

\begin{tabular}{|l|c|c|c|}
\hline & Adecuada & Parcialmente adecuada & Inadecuada \\
\hline Pre-test & 8 & 38 & 54 \\
\hline Pos-test & 54 & 31 & 15 \\
\hline
\end{tabular}

\section{Asimilación cognitiva sobre un hito histórico de América Latina}

Esta es otra de las variables que obtiene unos porcentajes muy bajos, pues las respuestas inadecuadas en ambas ensayos se sitúan entre el $85 \%$ y el $62 \%$, aunque hay que señalar que se produce un crecimiento de 30 puntos en las respuestas adecuadas. Sin embargo, a pesar de ello el dato ofrecido por la prueba $t(0.089)$ indica una ausencia de cambios significativos.

Tabla 35: Distribución porcentual sobre el conocimiento de un hito histórico de América Latina contemporánea (UDA)

\begin{tabular}{|l|c|c|c|}
\hline & Adecuada & Parcialmente adecuada & Inadecuada \\
\hline Pre-test & 8 & 8 & 85 \\
\hline Pos-test & 38 & 0 & 62 \\
\hline
\end{tabular}

\section{Asimilación cognitiva de un destacado pensador latinoamericano}

Este indicador en la fase del pre-test refleja que un $31 \%$ responden adecuadamente, frente a un $69 \%$ inadecuado. Sin embargo a pesar del crecimiento de 39 puntos en las respuestas adecuadas en el cuestionario final del semestre sigue manteniendo un elevado porcentaje, un $23 \%$. En cualquier caso ello no es obstáculo para que el resultado de la prueba $t(0.008)$ indique un avance sustancial y significativo en este conocimiento.

Tabla 36: Distribución porcentual sobre el conocimiento de un destacado pensador/a Latinoamericano (UDA)

\begin{tabular}{|l|c|c|c|}
\hline & Adecuada & Parcialmente adecuada & Inadecuada \\
\hline Pre-test & 31 & 0 & 69 \\
\hline Pos-test & 77 & 0 & 23 \\
\hline
\end{tabular}




\section{Asimilación cognitiva sobre un movimiento social en América Latina}

Los resultados obtenidos en el pre-test reflejan cómo un $23 \%$ responden adecuadamente mientras que un $15 \%$ son parcialmente adecuadas y un $62 \%$ inadecuadas. A pesar del crecimiento en las respuestas adecuadas, 23 puntos, del descenso de 7 puntos en las parcialmente adecuadas y de 16 puntos en las inadecuadas no llegan a producir un cambio significativo al ofrecer la prueba $t$ un índice de 0.209 .

Tabla 37: Distribución porcentual sobre el conocimiento de un movimiento social destacado en América Latina (UDA)

\begin{tabular}{|l|c|c|c|}
\hline & Adecuada & Parcialmente adecuada & Inadecuada \\
\hline Pre-test & 23 & 15 & 62 \\
\hline Pos-test & 46 & 8 & 46 \\
\hline
\end{tabular}

Práctica y acción: Sistematización de una experiencia docente desde la investigación social en la UNAE

Los datos resultantes del pre-test, sobre el total de los elementos conceptuales de la asignatura, contemplados en el módulo de conocimientos previos, revelaron en el momento inicial del semestre la constatación de una carencia significativa en el dominio conceptual por parte del alumnado. Ese indicador nos llevó a adoptar una intervención que tenía como principal orientación nivelar y reforzar la asimilación conceptual partiendo desde la práctica hasta la teoría a través de una propuesta de investigación acción. En ese sentido se concibió una intermediación y acompañamiento del docente direccionado a través de ejercicios prácticos y estímulo a potenciar los hábitos y prácticas culturales. Desde esa perspectiva se proponía inicialmente a los alumnos la realización de una actividad de iniciación a la investigación social, que sería el eje central de la materia y del propio proceso de aprendizaje, consistente en un "proyecto de aula", que combinaba una participación individual y trabajo colaborativo, con el propósito de reconstruir micros relatos de historias de vida centrados en el ámbito educativo histórico ecuatoriano.

Este proyecto de aula se definía, con respecto al sujeto a investigar, desde una doble perspectiva: rescatar la memoria de los maestros/as y de los alumnos en distintos momentos históricos. De ese modo se fijaba tres marcos temporales con el objeto de abarcar tres generaciones de estudio: la de los abuelos, los padres y los hermanos mayores. Ese planteamiento intentaba, además, dar cumplimiento al núcleo problemático, correspondiente al II Ciclo de Educación Básica, establecido en su malla curricular: escuela, familia y contexto.

Desde un plano metodológico se diseñaron varias fases de actuación tanto dentro como fuera del aula. Una primera estaba dedicada a caracterizar las nociones generales sobre la materia y los componentes esenciales que conforman el desarrollo de un proyecto de investigación en una fase de iniciación o aproximación. Esas sesiones fueron realizadas 
a través de un sistema de clases abiertas, de dialogo, debates y reflexiones a partir de lecturas básicas previas. Esto facilitó al alumnado familiarizarse con el trabajo, ejercitarse en casos concretos y en el uso de las técnicas de entrevistas, que se llevó a cabo a través de un proceso de estudio y elaboración bajo tres modalidades: a) individual, b) grupal; y c) colectiva.

Una segunda etapa fue la celebración de un curso de capacitación sobre el uso de bases de datos digitales bibliográficas, que sería reforzado, posteriormente, con una jornada de trabajo en la biblioteca universitaria con la tarea de recopilar bibliografía específica sobre historias de vida.

Una tercera etapa se dedicó al estudio y análisis específico del índice de una tesis doctoral $^{3}$, centrada en historias de vida de maestros y maestras, con la finalidad de un acercamiento al proceso estructural científico y en la elaboración de un microrelato de historia de vida del alumno en su contexto educativo.

Desde otra vertiente, encaminada a fomentar hábitos y prácticas culturales, se encargaba a los alumnos de ambos paralelos la organización, programación y participación en una actividad denominada "Kallpay Yachana o Competencia de Saberes", que consistía en una competición de conocimientos teóricos y prácticos sustentados en cuatro elementos claves: poner a prueba a los alumnos sobre el dominio de contenidos y procedimientos de la materia "Investigación y acción participativa: Historias de Vida"; estimular y fomentar el espíritu creativo de los estudiantes a través de la elaboración de distintas expresiones y manifestaciones culturales, cuyo motivo central debía basarse en la utilización de los contenidos y procedimientos significativos de la asignatura con la intención de reforzar su aprendizaje y asimilación de conocimiento tanto de forma individual como colectiva mediante esta socialización; estrechar lazos de convivencia y vinculación entre la comunidad universitaria UNAE, la sociedad azogueña y ecuatoriana en general; y aplicar una estrategia didáctica y de investigación como ejercicio de reflexión y autocrítica de la práctica docente.

En definitiva, desconocemos el nivel de incidencia que este planteamiento pudiera haber ejercido en los resultados obtenidos con respecto a la aplicación de éste u otros recursos o modelos. Pero lo que no cabe duda es que los datos proporcionados en el pos-test, como hemos vistos anteriormente, reflejan un cambio altamente significativo en todas las variables relacionadas con el módulo de asimilación de conocimientos. Esa dinámica puede tener como lectura que ese aprendizaje significativo alcanzado por el alumnado podría responder al modelo constructivista conceptual aplicado: desde la propia experiencia práctica, tanto individual como colaborativa, acompañado con espacios de reflexión, análisis y debates, que facilitaron un dominio y asimilación conceptual teórica significativa.

\section{Práctica y acción: Sistematización de la experiencia docente en la U. Cuenca}

El pre-test realizado mostró en el módulo de conocimientos previos, la carencia del manejo conceptual de la asignatura por parte de los estudiantes, además del desconocimiento de

Leite Méndez, A. E. (2011). Historias de vida de maestros y maestras. La interminable construcción de las identidades: vida personal, trabajo y desarrollo profesional. Tesis Doctoral dirigida por Ángel I. Pérez Gómez. Servicio publicaciones Universidad de Málaga. Disponible en: http://riuma.uma.es/xmlui/bitstream/handle/10630/4678/TDR_LEITE_MENDEZ.pdf 
los instrumentos normativos institucionales existentes en el área de conocimiento. A partir de los datos obtenidos, se resolvió desarrollar y fortalecer el nivel teórico conceptual de la asignatura. De esta forma, se definió establecer los elementos teóricos conceptuales nucleares como punto de partida y de desarrollo a lo largo de todo el abordaje de la asignatura, para terminar con una construcción teórico-conceptual propia de parte de los estudiantes.

Se propuso para ello, interrelacionar el tema inicial sobre Ética y Moral con la práctica en la formación universitaria y su proyección en el ejercicio profesional. La estrategia metodológica planteada, llevó a establecer varias fases que incluyeron el trabajo individual y grupal con la facilitación docente. La primera, de aproximación conceptual general, se dio a partir de clases que incluyeron la exposición y debate, de consultas y de sistematización grupal en un informe síntesis sobre las relaciones entre ética y moral.

La segunda fase, se realizó en grupos de trabajo para analizar el Código de Ética de la Universidad, su vigencia y cumplimiento a partir de las experiencias de los estudiantes a lo largo de su vida estudiantil, cuyos resultados se presentaron en una jornada de socialización. Se procedió luego a retroalimentar los elementos conceptuales iniciales.

La tercera fase consistió en realizar una búsqueda de documentación audiovisual sobre experiencias de aplicación de la ética en la profesión, la misma que fue complementada en algunos casos, con diálogos con profesionales del área. A partir de esta actividad se desarrolló una exposición grupal sobre la ética profesional y su importancia.

Cada una de las fases y de las actividades realizadas, permitieron una aproximación a la temática de estudio desde la práctica estudiantil, si bien no se puede afirmar explícitamente la incidencia que tuvieron en la asimilación teórico-conceptual, el logro de aprendizajes clave se potencia a través de la integración a su experiencia estudiantil. Los resultados del post-test nos permiten afirmar que se ha dado un cambio significativo en las variables referidas al módulo de asimilación de conocimientos, básicamente a partir del modelo constructivista que ha estado como elemento subyacente a lo largo de todo el proceso desarrollado activamente a través de los procesos cognitivos generados.

\section{Práctica y acción: Sistematización metodológica de la experiencia docente en la UDA}

La propuesta metodológica para abordar las temáticas en torno a América Latina su historia y la comprensión de la realidad actual, parte de la necesidad de generar pensamiento crítico como una prioridad para la formación de futuros docentes desde un saber situado. En este sentido se trabajó permanentemente en la lectura de autores latinoamericanos para la elaboración de análisis críticos que se realizaron de forma conjunta para aprender diversas metodologías en la elaboración de informes que luego se ponían en debate en clase. De esta manera la reflexión crítica y permanente basada en una relación dialógica se convierte en una metodología de enseñanza y experiencia propia para las sesiones prácticas que desarrollan durante el curso en el área de Ciencias Sociales.

Paralelamente al desarrollo de la parte teórica, se aplicó el estudio del cine documental histórico, esto mediante la elección de películas latinoamericanas que han tratado de forma crítica: situaciones, hechos o períodos clave para la comprensión de la realidad de nuestra región, se utilizó una guía de análisis interdisciplinario para situar: los hechos, los discursos, las narrativas, el pensamiento, etc. en el cine como instrumento didáctico para el aprendizaje de la historia. 
Para el abordaje de las temáticas actuales se realizó una puesta en común de las problemáticas que el alumnado conoce y le interesa profundizar, o sobre temáticas nuevas propuestas en el curso. La participación de los y las estudiantes tuvo una parte de trabajo individual y la otra fue una construcción colectiva. Se definieron las problemáticas de acorde al interés de cada grupo y se procedió a establecer una guía común para la investigación. De manera conjunta y con la mediación de la profesora se estableció una línea crítica de lectura de la realidad actual de nuestra región, entendiendo tal como lo plantea Bell Hooks al aula como un espacio de transformación de pensamiento, para desnaturalizar las desigualdades que nos afectan en América Latina. Los materiales de investigación fueron diversos: material bibliográfico; cine documental; hemeroteca; entrevistas en medios digitales y escritos, entrevistas personales, así como trabajo de campo que fue aplicado por uno de los grupos. Una vez que cada grupo desarrolló la investigación, los resultados fueron expuestos en el aula aplicando diversas estrategias didácticas y al final cada alumna y alumno debían responder a la pregunta de ¿Cómo piensan la problemática que investigaron y si investigarla les hizo plantearse nuevas cuestiones y nuevas formas de ver nuestra región?

\section{CONCLUSIONES}

El perfil del alumnado de las tres universidades presenta determinadas características similares: la mayor parte de los mismos proceden del espacio territorial del entorno cercano donde se ubican los centros; se observa que las edades de los estudiantes, en relación al ciclo semestral que cursan, son elevadas; se manifiesta un equilibrio proporcional de género entre los estudiante, aunque con una mayor presencia de mujeres en la UDA; la mayor parte de los alumnos de la UNAE y la UDA provienen de centros educativos fiscales y se registra un equilibrio entre centros fiscales y particulares en los estudiantes de la U. de Cuenca; un gran porcentajes de estudiantes de las tres universidades se encuentra en un estado civil de soltería, no obstante un $10 \%$ cuentan con cargas familiares; y éticamente se verifica un hegemonía estudiantil autodefinida como mestiza.

Un segundo aspecto detectado evidencia la existencia de un alto grado de madures motivacional a la hora de elegir sus carreras, al tiempo que manifiestan unas definidas expectativas sobre las materias y una valoración positiva junto a una fuerte carencia de los modelos pedagógicos de sus centros universitarios.

Otro rasgo característico, referido al estado previo sobre la base cognitiva y científica, es la dificultad que presentan los alumnos analizados en la comprensión y aplicación de conocimientos científicos básicos. En conjunto se puede decir que los porcentajes de respuestas adecuadas referidas al módulo conceptual en el pre-test ofrecen unos índices bajos. En cambio esa situación, tras la fase final del proceso de aprendizaje semestral, experimenta una notable transformación, reflejada en un avance sustancial significativo en el dominio conceptual y de conocimiento sobre de la materia.

Esa evolución indica que, al menos, se ha producido un aprendizaje de conocimiento a lo largo del semestre y, por tanto, la labor realizada por los docentes a través de los distintos planteamientos didáctico-práctico diseñados junto a las acciones de estímulos aplicadas han podido ejercer alguna incidencia en ese proceso de aprendizaje, sin que se pueda precisar con claridad su dimensión, aunque también ha llevado a la conclusión de 
profundizar en esta experiencia docente en ciclos posteriores. Por tanto, independiente del planteamiento estratégico docente empleado se constata asimismo una dinámica de crecimiento porcentual de aprendizaje y cambio significativo, aunque con ritmos diferenciados entre las universidades.

En definitiva, indagar sobre los conocimientos previos del alumnado al iniciar una materia o bien en una nueva etapa formativa, como chequear su evolución, es un modo de aproximación al estado real de sus conocimientos que proporciona información muy relevante al docente para encauzar estrategias didácticas y diseñar acciones prácticas, además, de constituir una herramienta de autoevaluación, reflexión metodológica, revisión de estrategias, planteamientos didácticos y cambio de prioridades en los objetivos de la enseñanza, junto a otras maneras de concebir la relación profesor-alumno, de potenciar su papel de acompañamiento e innovar en la organización del tiempo y los espacios, además, de la incorporación de elementos culturales que potencien una formación integral y significativa a partir de la construcción de conocimientos propio de la materia. Y por último esta experiencia docente interuniversitaria ha servido a sus autores para reflexionar sobre su desempeño y superar las fronteras del aula para conectar con otros espacios y acciones que han generado un enriquecimiento compartido a través de este proceso colaborativo.

\section{REFERENCIAS BIBLIOGRÁFICAS}

Bonilla Marchán, A., Delgado, R. \& Stefos, E. (2017). The Social Characteristics of Postgraduate Students in Ecuador: A Multidimensional Statistical Analysis. Review of European Studies, Vol. 9, No. 2; 2017. pp. 35-44. doi:10.5539/res.v9n2p35

Carretero, M. \& Limón M. (1997). Problemas actuales del constructivismo. De la teoría a la práctica. En La construcción del conocimiento escolar. Paidós, pp. 137-154. Recuperado el 15 de julio de 2017 desde https://studylib.es/doc/299607/carretero-m--lim\%C3\%B3n-m.-problemas-actuales-del

Coll, C., Martín, E., Mauri, T. Miras, M., Onrubia, J., Solé, I., \& Zabala, A. (2004). El constructivismo en el aula. Barcelona. Graó.

García-Carmona, A., \& Acevedo Díaz, J. A. (2016). Concepciones de estudiantes de profesorado de educación primaria sobre la naturaleza de la ciencia: Una evaluación diagnóstica a partir de reflexiones en equipo. Revista mexicana de investigación educativa, 21(69), pp. 583-610. Recuperado el 15 de julio de 2017 desde http://www.scielo.org.mx/scielo.php?pid=S1405$66662016000200583 \&$ script=sci_arttext

Kampouropoulou-Savvaidou, M., Efstathiou, I. \& Stefos, E. (2014). Aplicaciones Educativas de Arte y de Patrimonio Cultural. Evaluación de tareas como manuales educativos. p.464. Atenas: Publicaciones Diadrasi.

Limón, M. \& Carretero, M. (1996). Las ideas previas de los alumnos: ¿Qué aporta este enfoque a la enseñanza de las Ciencias? En Construir y enseñar: las Ciencias Experimentales. Aique, Buenos Aires.

López Recacha, J. A. (2009). La importancia de los conocimientos previos para el aprendizaje de nuevos contenidos. Disponible en: http://www.csi-csif.es/andalucia/modules/mod_ense/revista/ pdf/Numero_16/JOSE\%20ANTONIO_LOPEZ_1.pdf

Martínez, J. L. (2015). Evaluación diagnóstica de conocimientos científicos en dos cursos de educación secundaria mediante un mismo instrumento de autoevaluación. Revista de la Asociación Colombiana de Ciencias Biológicas, (24), pp.90-96. Recuperado el 15 de julio de 2017 desde Recuperado el 15 de julio de 2017 desde http://www.ojs. asociacioncolombianadecienciasbiologicas.org/index.php/accb/article/view/79 
Moreira, M. A. (2008). Organizadores previos y aprendizaje significativo. Revista Chilena de Educación Científica, 7(2), 23-30. Recuperado el 15 de julio de 2017 desde https://www.if.ufrgs. br/ moreira/ORGANIZADORESesp.pdf

Novack, J.D. (1987). Constructivismo humano: un consenso emergente. Enseñanza de las Ciencias, 6(3), 213-223. Recuperado el 15 de julio de 2017 desde https://ddd.uab.cat/pub/ edlc/02124521v6n3/02124521v6n3p213.pdf

Papantonakis, G., Athanasiadis, I. \& Stefos, E. (2010). La comprensión del texto literario por los estudiantes de los dos últimos cursos de la Educación Primaria en las escuelas de Grecia. Nea Pedia, 135. pp. 79-95. Athens: Patakis.

Papapostolou, I. \& Stefos, E. (2013). Qualitative analysis on pedagogical research. Methodological approaches. In I. Papapostolou (Ed.), Educational activities. Teaching Interventions in Secondary Education. pp. 244-251. Rhodes: Evdimos Editions.

Papapostolou, I., Papapostoulou, K. \& Stefos, E. (2013). Educational Research. From Qualitative to Quantitative analysis. p.178. Rhodes: Evdimos Editions.

Pimienta Prieto, J. H. (2012). Estrategias de enseñanza-aprendizaje. Docencia universitaria basada en competencias. Ed. Pearson Educación. México.

Rayas, J. (2004). El reconocimiento de las ideas previas como condición necesaria para mejorar las posibilidades de los alumnos en los procesos educativos en ciencias naturales. Revista Xictli de la Unidad UPD, 94. Recuperado el 15 de julio de 2017 desde https://docs.google.com/ document/d/1poF4K3A0eOPUvkAvqAMdWXOE-sV-LUPe85kNUaX7WGg/edit

Restrepo Gómez, B., Román Maldonado, C., \& Londoño Giraldo, E. (2014). Evaluación diagnóstica inicial en programas de educación superior virtual de la Católica del Norte Fundación Universitaria. Estudio cuasiexperimental Colombia. Revista Virtual Universidad Católica del Norte, No. 34. 60-77. Recuperado el 15 de julio de 2017 desde http://200.24.17.68:8080/jspui/ bitstream/123456789/218/1/Bernardo\%20Restrepo.pdf

Solaz-Portolés, J. J. \& Sanjosé López, V. (2008). Conocimiento previo, modelos mentales y resolución de problemas. Un estudio con alumnos de bachillerato. Revista electrónica de investigación educativa, vol.10 no. 1. Recuperado el 15 de julio de 2017 desde http://www.scielo.org.mx/ scielo.php?pid=S1607-40412008000100004\&script=sci_arttext

Stefos, E. \& Efstathiou, I. (2013). Quantitative analysis of the data of the School of Trianta during the period of 1906-1916. In I. Papapostolou (Ed.), Educational activities. Teaching Interventions in Secondary Education. pp. 29-57. Rhodes: Evdimos Editions.

Stefos, E. \& Koulianidi, G. (2016a). Nutrition Data Analysis Using R: Applications in Higher Education. Health Sciences Research. Vol. 3, No. 1, 2016, pp. 10-16.

Stefos, E. \& Koulianidi, G. (2016b). El uso del software R en la enseñanza de Estadística. Aplicaciones en la Pontificia Universidad Católica del Ecuador. En F. Rivas Echeverría, S. Arciniegas Aguirre, Avances y aplicaciones de sistemas inteligentes y nuevas tecnologías. pp. 57-65. Mérida, Venezuela: Coeditado por Consejo de Publicaciones de la Universidad de los Andes \& PUCE-SI.

Stefos, E. \& Papapostolou, I. (2013). Research Methodology. Processes and suggestions. p. 406. Rhodes: Evdimos Editions.

Stefos, E., Athanasiadis I., Gialamas, B. \& Tsolakidis, C. (2011). El uso de las Nuevas Tecnologías y el Método de Proyectos en la enseñanza de Estadística: Un estudio de caso en la Educación Superior. HMS i JME, Volume 3. 2010-2011. pp.84-100.

Villegas, D. J. M., \& Pereira, R. E. V. (2015). Papel de los conocimientos previos en el aprendizaje de la matemática universitaria. Acta Scientiarum. Education, 37(1), 85. Recuperado el 15 de julio de 2017 desde http://search.proquest.com/openview/dcb3b4ec170857dfefc06e147cc36a 8 $1 / 1$ ?pq-origsite $=$ gscholar\&cbl $=2037656$ 